\title{
ADAPTACIÓN DE LA METODOLOGÍA TUNING PARA EL LEVANTAMIENTO DEL PERFIL DE EGRESO DE LOS ALUMNOS DE BACHILLERATO DE LA UNIVERSIDAD DE LOS ANDES
}

\author{
Juan Carlos Aguilera ${ }^{1}$, Gabriela Cuevas ${ }^{2}$
}

\section{RESUMEN}

El objetivo del presente trabajo consiste en dar a conocer, de manera descriptiva, el modo en que se realizó el levantamiento del perfil de egreso de los alumnos del Programa de Bachillerato de la Universidad de los Andes (Chile), utilizando una adaptación de la metodología que se deriva del Proyecto Tuning y del Proyecto Tuning para Latinoamérica y las directrices para la elaboración de perfiles de egreso institucional.

Considerando que el Programa de Bachillerato tiene un carácter propedéutico a los estudios universitarios, este trabajo constituye una novedad al adaptar una metodología orientada a la elaboración de perfiles de carreras con salida profesional.

Palabras clave: participación, habilidades (competencias), perfil de egreso, programa bachillerato.

\section{ADAPTATION OF TUNING METHODOLOGY FOR IMPROVING THE GRADUATION PROFILE OF BACHELOR'S DEGREE IN UNIVERSIDAD DE LOS ANDES}

\section{ABSTRACT}

This paper is aimed to present in detail a survey it conducted on the graduation profile of students in the Baccalaureate Program of the Universidad de los Andes (Chile), using an adaptation of the methodology deriving from the Tuning Project and the Tuning Project for Latin America and the Guidelines for the profile forming of graduation from the Universidad de los Andes (CHILE). Considering that Baccalaureate Program has a preparatory character for university studies, this work is an innovation since it adapts a methodology aimed to develop career profiles with professional output.

Keywords: participation, skills, profile of expenditure, baccalaureate programs.

1 Director de Estudios. Programa Bachillerato, Universidad de los Andes, Santiago, Chile.

2 Directora. Programa Bachillerato, Universidad de los Andes, Santiago, Chile. Contacto: jcaguilera@uandes.cl 


\section{ADAPTACIÓN DE LA METODOLOGÍA TUNING PARA EL LEVANTAMIENTO DEL PERFIL DE EGRESO DE LOS ALUMNOS DE BACHILLERATO DE LA UNIVERSIDAD DE LOS ANDES}

\section{Introducción}

Uno de los proyectos relevantes de educación superior en Europa, y que se ha ido extendiendo a América Latina, es el Tuning Educational Structures in Europe. Los responsables de su diseño eligieron el vocablo to tune -que remite al significado lingüístico afinar-, para transmitir la idea de que las universidades buscan llegar de manera conjunta y armónica a puntos de referencia comunes, basados en el respeto a las peculiaridades de cada proyecto universitario y fundados en la confianza mutua. El tiempo verbal en que está expresado el vocablo -gerundio-, pone de manifiesto la idea de que es algo que está en proceso y siempre lo estará, en virtud de que la educación debe mantenerse en diálogo con las necesidades sociales. Es decir, el antecedente que se tiene en cuenta consiste en que un área social y económica debe ir en paralelo a un área de educación. La cuestión de fondo es generar un "Espacio Europeo de Educación Superior" (EEES) al año 2010. Con este propósito se propone desarrollar cuatro líneas de acción: a) competencias genéricas, b) competencias específicas de las áreas temáticas, c) papel del sistema ECTS como sistema de transferencia y acumulación de créditos, y d) enfoque de aprendizaje, enseñanza y la evaluación en relación con la garantía y control de calidad. En América Latina, el Proyecto Alfa Tuning, tiene similar finalidad y, siguiendo su propia metodología, tiene cuatro grandes líneas: a) competencias (genéricas y específicas de las áreas temáticas), b) enfoques de enseñanza, aprendizaje y evaluación, c) créditos académicos y d) calidad de los programas.

Un trabajo serio y articulado en las cuatro líneas propuestas incidirá en el fomento de la transparencia de los perfiles profesionales y académicos. Además, el interés en el desarrollo de habilidades y la 
utilización del sistema de créditos, centran la educación en el alumno y en su capacidad de aprender, exigiendo mayor protagonismo y compromiso.

En concordancia con la metodología elegida para llevar adelante nuestro empeño, hay que considerar que uno de los aspectos fundamentales de la universidad del siglo XXI, inserta en la llamada sociedad de la información, consiste en que el conocimiento entendido como un bien compartible -mientras más se comparte más crece-, solamente puede lograrse en comunidades de investigación y aprendizaje. Así, la necesidad de recurrir a quienes poseen el saber resulta imperiosa; tal como lo vio Aristóteles, el protagonista nato de la educación es el estudiante, no el profesor iluminado. Por consiguiente, el diálogo, es decir, la participación fundada en el saber y en el respeto de la dignidad de la persona, son los modos naturales de convivencia en la llamada casa del saber humano.

En virtud de tales consideraciones, se realizó el trabajo de levantamiento del perfil de egreso de los alumnos del Programa de Bachillerato de la Universidad de los Andes, utilizando una metodología que, en cierto sentido, tuviera en cuenta ese modo de convivir en la institución.

La importancia que tiene dar a conocer el presente trabajo a la comunidad académica reside en que utilizando una metodología que no fue diseñada para la elaboración de perfiles de egreso de programas sin salida profesional, como es el caso del Bachillerato, se ha podido adaptar y utilizar de manera innovadora, contando con la participación y compromiso de autoridades académicas, docentes y alumnos de la totalidad de las carreras de la universidad. Ha generado, en cierto sentido, un espacio común de discusión, análisis y colaboración, cuestión de la máxima importancia, si se considera los, a veces, atomizados esfuerzos que se realizan en las facultades para que se relacionen las diversas áreas del conocimiento, quedando como una promesa incumplida la tan necesaria interdisciplinariedad. 
222 ADAPTACIÓN DE LA METODOLOGÍA TUNING PARA EL LEVANTAMIENTO DEL PERFIL DE EGRESO DE LOS ALUMNOS DE BACHILLERATO DE LA UNIVERSIDAD DE LOS ANDES J.C. Aguilera, G. Cuevas

\section{Objetivo}

El objetivo del presente trabajo consiste en dar a conocer de manera descriptiva el modo en que se realizó el levantamiento del perfil de egreso de los alumnos del Programa de Bachillerato de la Universidad de los Andes (Chile), utilizando una adaptación de la metodología que se deriva del Proyecto Tuning y del Proyecto Tuning para Latinoamérica y las directrices para la elaboración de perfiles de egreso institucionales.

Con la finalidad de acotar mejor el objetivo del presente trabajo, hay que mencionar que por el carácter descriptivo de la experiencia que se da a conocer, esta no pretende argumentar ni sostener cómo y porqué el Espacio Europeo de Educación Superior (EEES) debería replicarse en Chile, o si sería una experiencia que debería imitarse. Tampoco se intenta analizar la importancia o no de la aplicabilidad de modelos foráneos al sistema chileno, ni proponer un elenco de competencias para el nivel propedéutico (bachillerato) de la formación universitaria chilena o una metodología de participación y consulta de los actores universitarios en los procesos de evaluación, y menos tomar partido ideológico respecto del Proyecto Tuning. Tales propósitos exceden ampliamente el objetivo del presente trabajo, a saber: describir la labor realizada en el levantamiento del perfil de egreso de los alumnos del Programa de Bachillerato de la Universidad de los Andes.

\section{Método y resultados}

Con el propósito de revisar la efectividad, validez, pertinencia y actualización del Programa de Bachillerato de la Universidad de los Andes, después de cinco años de existencia, en septiembre de 2009, se llevó a cabo una "Jornada de Análisis y Planificación", que contó con la participación de autoridades académicas y administrativas, profesores del Programa, expertos en educación superior y en programas propedéuticos y los miembros que conforman el Consejo Directivo del Programa de Bachillerato. Las conclusiones se resumieron en un documento que fue evaluado por la rectoría, coincidiendo con las conclusiones del informe respecto de la necesidad de estudiar una 
reformulación del Programa, recomendando que para tal propósito se considerara el aporte de las autoridades y diversas unidades académicas de la universidad.

Con tales antecedentes, en enero del 2010, se determinó utilizar una metodología de trabajo que estuviera validada en el mundo universitario y de acuerdo a los antecedentes que se reunieron en tal sentido. El Proyecto Tuning (Tuning Educational Structures in Europe, 2006), el Proyecto Alfa Tuning América Latina (2007) y el documento Metodología para el Levantamiento y Revisión de Perfiles de Egreso de la Universidad de los Andes (2008), cumplían con tales requisitos. Luego, teniendo en cuenta las metodologías propuestas en los documentos citados, se organizó el trabajo de reformulación del programa en torno a dos temas centrales: definición del perfil del egresado del programa de bachillerato y diseño del plan de estudios de acuerdo al perfil.

La metodología propuesta en los proyectos Tuning Europa y Latinoamérica está concebida para el diseño de perfiles de egreso de carreras universitarias o técnicas con salida profesional. Por tal motivo, considerando que el programa de bachillerato tiene un carácter propedéutico a los estudios universitarios y la salida de los egresados se orienta a una carrera universitaria, se realizó una adaptación de tal modelo para los propósitos de nuestro trabajo. Si la elaboración de perfiles de egreso para carreras con salida profesional contempla la participación de profesionales egresados, empleadores y académicos, en el caso particular de este Programa se consideró como estudiantes graduados a quienes están cursando segundo y tercer año de una carrera en la universidad, los empleadores se homologaron a los directores de estudios y decanos de la totalidad de las carreras que imparte la universidad y fueron considerados académicos aquellos que imparten alguna asignatura mínima, en el primer año de estudios, de alguna de las carreras de la institución. De acuerdo a la definición de la universidad, que distingue tres áreas de conocimiento: Humanidades, Administración y Ciencias de la Salud, en las que se incardinan las carreras, se conformaron paneles de expertos, profesores y alumnos. Además, se utilizaron las clasificaciones de competencias descritas en los proyectos Tuning tanto europeo como para Latinoamérica 
y el documento de la Universidad de los Andes que propone la metodología para el levantamiento de los perfiles de egreso. De manera que el criterio para la clasificación de las competencias que se utilizó resulta de la clasificación presente en los documentos citados que, en lo sustancial, no varía.

De acuerdo a la metodología elegida, participaron en el desarrollo del trabajo 36 profesores, 24 alumnos, 14 directores de estudios, decanos de algunas de las facultades y 6 profesores, que ayudaron en la revisión de la redacción de los cuestionarios que se elaboraron. Para la elección de los participantes se utilizaron los siguientes criterios: en el caso de los profesores, se solicitó, vía correo electrónico y luego telefónicamente, a los directores de Estudio los nombres de los profesores más destacados de asignaturas de carreras del primer año. En el caso de los alumnos, se determinó que deberían provenir de las diferentes carreras que imparte la universidad, que estuvieran cursando segundo o tercer año y hubieran ingresado directamente o vía bachillerato, con rendimiento regular (bajo 5,5) y bueno (igual o superior a 6). Determinado el perfil, se eligieron aleatoriamente. En el caso de los decanos, se trabajó con aquellos que dirigen las carreras que reciben mayor número de alumnos provenientes del Programa de Bachillerato.

Definidos los nombres de los decanos, profesores y alumnos, se elaboraron listas de acuerdo a las diferentes áreas de conocimiento definidas por la universidad, con nombres, direcciones electrónicas y teléfonos celulares de profesores y alumnos. Luego, se les invitó -mediante correo electrónico y telefónicamente- a participar en un panel de expertos. Por otra parte, se elaboró un guión de entrevista que permitiera dirigir las sesiones de dichos paneles.

Se llevaron a cabo tres paneles en los que participaron profesores y tres en los que participaron alumnos, con la asistencia de la totalidad de los profesores y alumnos invitados. En todos los casos, las sesiones fueron grabadas con la anuencia de los asistentes, se tomó nota por parte de un secretario, y se redactó un acta de cada sesión. Al día siguiente a las sesiones, se envió un correo electrónico para agradecer la participación y posteriormente se entregó un cuestionario breve por 
si deseaban agregar alguna idea que no alcanzaron a desarrollar en la sesión, y para que nombraran tres hábitos, competencias, que deberían poseer los alumnos de primer año de carrera para tener un desempeño académico sobresaliente. Además, se envió un correo electrónico a la totalidad de los directores de Estudio de las diferentes carreras de la universidad para informar del trabajo realizado y agradecer el apoyo brindado en la elaboración de las listas de los paneles de expertos.

Posteriormente, se sintetizó la información contenida en las actas, las grabaciones y cuestionarios breves y se elaboró una lista preliminar de habilidades (competencias), de acuerdo al programa Tuning para Europa, el de América Latina y el documento para el levantamiento de perfiles de egreso de la universidad, las que se agruparon en: a) competencias alumnos de primer año: generales básicas (7), instrumentales (12), interpersonales (4), sistemáticas (6), y b) específicas de bachillerato (22) (ver anexo 1 tabla de competencias). A continuación, se solicitó a seis profesores que revisaran críticamente el documento, especialmente con relación a la claridad y redacción de las habilidades; hubo algunas observaciones respecto de la redacción y ubicación de algunas habilidades que se determinaron genéricas y quedaban mejor como instrumentales. Luego, se envió vía correo electrónico e impreso el documento a todos los directores de Estudio, profesores y alumnos que conformaron los paneles con el propósito de que jerarquizaran, poniendo nota de 1 a 7 a cada una de las diferentes habilidades (competencias). Una vez recogida toda la información se procedió a tabularla considerando el origen de la información, -directores de estudio, profesores y alumnos-, luego se sumó el puntaje otorgado a cada habilidad y se procedió a jerarquizar por puntaje obtenido.

La jerarquización de los resultados encontrados, sintéticamente, fueron los siguientes.

Para las denominadas competencias alumnos de primer año:

a) Generales básicas: la que resultó con mayor puntaje fue responsabilidad personal, seguida de desarrollo correspondiente a la edad de la memoria intelectual, y de interés por aprender.

b) Instrumentales: la mayor calificación la obtuvo la capacidad de 
precisión en la expresión oral o escrita de las propias ideas o ajenas. A continuación se ubicaron con idéntico puntaje: capacidad de redactar y expresar en forma oral o escrita, lógicamente, las ideas propias o ajenas, y capacidad de identificar, plantear y resolver problemas. Seguidamente, se ubicó la capacidad de abstracción, análisis y síntesis.

c) Interpersonales: capacidad de crítica y autocrítica, desarrollar habilidades para las relaciones interpersonales e inteligencia emocional y capacidad de trabajo en equipo, fueron las competencias que alcanzaron el primer, segundo y tercer mejor puntaje.

d) Sistemáticas: la capacidad de aprender fue la que obtuvo el mejor puntaje, seguida de motivación por la calidad e iniciativa.

Para las competencias específicas de bachillerato:

Adquirir hábitos de trabajo bien hecho y capacidad de trabajo intenso y constante fueron las competencias que obtuvieron el mejor puntaje, seguidas de desarrollar el pensamiento lógico crítico y creativo, y en tercer lugar adquirir o mejorar habilidades de memoria, comprensión, abstracción, síntesis y análisis.

Una vez jerarquizadas las habilidades, se envió la lista con las que tuvieron mejor puntuación a los directores de estudio, responsables de liderar los cambios en los planes de estudio y a otros que tienen que ver con la marcha académica de las facultades, para que realizaran una nueva jerarquización que permitiría determinar las habilidades (competencias) que finalmente configurarían el perfil de egreso de bachillerato. Los resultados obtenidos fueron los siguientes:

Competencias básicas: responsabilidad personal. Instrumentales: capacidad de comunicación lógica y fluida, en los planos oral y escrito, de ideas propias y ajenas. Con relación a las competencias específicas de bachillerato: hábito de trabajo intenso y constante; desarrollo del pensamiento lógico, crítico y creativo.

Finalmente, en ésta primera parte del trabajo de definición del perfil de egreso de los alumnos del programa de bachillerato, se procedió a elaborar el mapa de habilidades y competencias. Este contempla la definición de la habilidad, criterios que la especifican, 
actividades que debería realizar el profesor para que se adquieran, actividades que deberían realizar los alumnos y, por último, los indicadores de evaluación.

Al final, se determinaron las asignaturas y los créditos que corresponderían a cada una de ellas, de acuerdo al Sistema de Créditos Transferibles-Chile (SCT-Chile).

La puesta en marcha del proyecto, no ofreció dificultades ni tampoco generó resistencia, prueba de ello es el buen logro de la convocatoria a participar. Pensamos que tal actitud fue por los medios y el modo en que se les invitó a participar, de manera afectiva y efectiva, por medio de correo electrónico y teléfono, y el que advirtieran que sus opiniones verdaderamente importaban. Además, explicarles el rigor metodológico que se utilizaría ayudó a generar discusiones de alto alcance que en este trabajo no es posible reflejar totalmente. Un problema técnico con las primeras grabaciones en que se utilizó cinta magnética y no disco digital, que hace más fácil la trascripción de las sesiones, pudo evitarse, ahorrando tiempo.

\section{Conclusiones}

La metodología utilizada, si bien corresponde a un proyecto ampliamente aceptado (Tuning) y orientado a las carreras con salida profesional, la adaptación a un programa con carácter propedéutico constituye un aporte innovador de experiencia académica universitaria.

La utilización de la metodología elegida resultó eficaz en el empeño por definir el perfil de egreso de los alumnos del programa de bachillerato. Además, permitió generar espacios de participación de los diferentes estamentos de la universidad y entre ellos.

Por otra parte, múltiples son los desafíos que actualmente se están enfrentando para llevar adelante el programa y posteriormente evaluar los resultados a corto y mediano plazo. En este sentido, la contratación de académicos con un perfil acorde; la planificación de las asignaturas de acuerdo a un nuevo modelo que incluye la planificación 
clase a clase y la bibliografía a utilizar por los alumnos; el diseño del trabajo que el profesor da al alumno de acuerdo al diccionario de competencias; el asesoramiento académico a los alumnos para que tengan claridad acerca del Programa; la capacitación de los asesores académicos para entrevistas con los alumnos y especialmente con relación a las horas de estudio que están contempladas para cada asignatura y la planificación estratégica del tiempo que eso implica; la utilización de la biblioteca por parte de los alumnos de acuerdo a la bibliografía especificada en los programas; la coordinación y seguimiento del trabajo de los profesores; el diseño de una carga balanceada y constante de las evaluaciones en las diferentes asignaturas conforme al sistema de créditos, y otros aspectos que son propios de la gestión académica, resultan desafíos fundamentales para que los alumnos, efectivamente, desarrollen las capacidades que se determinaron en el perfil de egreso.

Con todo, el trabajo universitario riguroso es posible de realizar con la participación activa de los miembros de la comunidad de investigación y aprendizaje. El empeño por elaborar un nuevo perfil de egreso para el Programa de Bachillerato de la Universidad de los Andes (Chile) es un ejemplo en tal sentido.

\section{Referencias bibliográficas}

Tuning Educational Structures in Europe (2006).

Disponible en http://tuning.unideusto.org/tuningeu/images/stories/ template/General_Brochure_Spanish_version.pdf.

Proyecto Alfa Tuning América Latina (2007).

Disponible en http://tuning.unideusto.org/tuningal/index.php

Universidad de los Andes (2008) Metodología para el Levantamiento y revisión de perfiles de egreso. Documento interno.

Guía Práctica para la instalación del Sistema de Créditos Transferibles-Chile. SCT-Chile.

Disponible en http://www.sct-chile.cl/download/Guia_Practica_SCT.pdf

Recibido: 03/11/2010

Aceptado: 19/05/2011 


\section{Anexo 1. Clasificación de competencias}

\begin{tabular}{|c|c|}
\hline $\begin{array}{l}\text { Competencias genéricas } \\
\text { generales básicas }\end{array}$ & Importancia \\
\hline responsabilidad personal & 1234567 \\
\hline capacidad de organización y planificación (disciplina) & 1234567 \\
\hline capacidad de aprendizaje autónomo y autorregulado & 1234567 \\
\hline \multicolumn{2}{|l|}{ instrumentales } \\
\hline capacidad para seguir instrucciones en el desarrollo de una actividad & 1234567 \\
\hline manejo del computador & 1234567 \\
\hline $\begin{array}{l}\text { capacidad de gestión de la información: habilidad para buscar, seleccionar y } \\
\text { analizar la información proveniente de diferentes fuentes }\end{array}$ & 1234567 \\
\hline capacidad de resolución de problemas & 1234567 \\
\hline capacidad para tomar decisiones & 1234567 \\
\hline capacidad para hablar en público & 1234567 \\
\hline capacidad de expresar con coherencia y validez la opinión de otro & 1234567 \\
\hline capacidad de redacción y de expresar lógicamente las ideas & 1234567 \\
\hline conocimiento básico de una segunda lengua (inglés) & 1234567 \\
\hline \multicolumn{2}{|l|}{ interpersonales } \\
\hline capacidad de crítica y autocrítica & 1234567 \\
\hline capacidad de trabajo en equipo & 1234567 \\
\hline habilidades en las relaciones interpersonales: inteligencia emocional & 1234567 \\
\hline $\begin{array}{l}\text { capacidad de comunicarse con expertos y desarrollar redes de contacto con la } \\
\text { realidad }\end{array}$ & 1234567 \\
\hline \multicolumn{2}{|l|}{ sistemáticas } \\
\hline capacidad de abstracción, análisis y síntesis & 1234567 \\
\hline liderazgo & 1234567 \\
\hline capacidad de adaptación a nuevas situaciones & 1234567 \\
\hline capacidad de generar nuevas ideas (creatividad) & 1234567 \\
\hline iniciativa & 1234567 \\
\hline motivación por la calidad & 1234567 \\
\hline pensamiento crítico & 1234567 \\
\hline \multicolumn{2}{|l|}{ específicas } \\
\hline promover el desarrollo de aprendizaje estratégico y autorregulado & 1234567 \\
\hline desarrollar el pensamiento lógico, crítico y creativo & 1234567 \\
\hline redactar correctamente documentos de uso frecuente en el contexto universitario & 1234567 \\
\hline comunicación oral fluida & 1234567 \\
\hline $\begin{array}{l}\text { adquisición de hábito de la lectura sistemático de manera comprensiva, analítica } \\
\text { y reflexiva }\end{array}$ & 1234567 \\
\hline adquirir o mejorar habilidades de memoria, comprensión, abstracción y síntesis & 1234567 \\
\hline aprender hábitos y técnicas de concentración & 1234567 \\
\hline análisis de textos & 1234567 \\
\hline $\begin{array}{l}\text { desarrollar la capacidad de comprender y relacionar las distintas etapas de la } \\
\text { historia }\end{array}$ & 1234567 \\
\hline $\begin{array}{l}\text { capacidad de buscar y seleccionar y analizar información proveniente de fuentes } \\
\text { diversas }\end{array}$ & 1234567 \\
\hline $\begin{array}{l}\text { desarrollar la capacidad de interpretar fuentes de información relacionadas al } \\
\text { tema del curso }\end{array}$ & 1234567 \\
\hline
\end{tabular}


230 ADAPTACIÓN DE LA METODOLOGÍA TUNING PARA EL LEVANTAMIENTO DEL PERFIL DE EGRESO DE LOS ALUMNOS DE BACHILLERATO DE LA UNIVERSIDAD DE LOS ANDES J.C. Aguilera, G. Cuevas

\begin{tabular}{|l|l|}
\hline $\begin{array}{l}\text { aplicar, analizar y sintetizar los conocimientos adquiridos por medio de la } \\
\text { elaboración de ensayos }\end{array}$ & 1234567 \\
\hline $\begin{array}{l}\text { promover, a través de los grandes temas de la filosofía, su directa vinculación con } \\
\text { el gobierno de la propia vida }\end{array}$ & 1234567 \\
\hline $\begin{array}{l}\text { formularse de modo personal y filosóficamente alguna de las grandes preguntas } \\
\text { que han inquietado al hombre a través de su historia }\end{array}$ & 1234567 \\
\hline $\begin{array}{l}\text { comprender la importancia del saber filosófico y la relevancia que ha tenido en la } \\
\text { historia del pensamiento y de los acontecimientos humanos }\end{array}$ & 1234567 \\
\hline $\begin{array}{l}\text { ser capaz de aplicar y plantear problemas distintos parta ser resueltos con las } \\
\text { herramientas adecuadas e interpretar el resultado }\end{array}$ & 1234567 \\
\hline
\end{tabular}

